\title{
Efficient vacuum-deposited perovskite solar cells with stable cubic $\mathrm{FA}_{1-\mathrm{x}} \mathrm{MA}_{\mathbf{x}} \mathrm{PbI}_{3}$
}

Lidón Gil-Escrig, ${ }^{\dagger}$ Chris Dreessen,${ }^{\dagger}$ Ismail Cihan Kaya, ${ }^{\dagger},{ }^{\dagger}$ Beom-Soo Kim,${ }^{\dagger}$ Francisco Palazon, ${ }^{\dagger}$ Michele Sessolo, ${ }^{\dagger}, *$ Henk J. Bolink, ${ }^{\dagger}$

†nstituto de Ciencia Molecular, Universidad de Valencia, C/ Catedrático J. Beltrán 2, 46980, Paterna, Spain Department of Metallurgical and Materials Engineering, Konya Technical University, Konya, Turkey *Corresponding author: michele.sessolo@uv.es 


\section{EXPERIMENTAL PROCEDURES}

\section{Materials}

N4,N4,N4 'N4 '-tetra([1,1'-biphenyl]-4-yl)-[1,1':4',1'-terphenyl]-4,4'-diamine (TaTm) was provided by Novaled $\mathrm{GmbH}$ and Fullerene $\left(\mathrm{C}_{60}\right)$ was purchased from Merck KGaA. $\mathrm{PbI}_{2}$, $\mathrm{CH}_{3} \mathrm{NH}_{3} \mathrm{I}$ (MAI), $\mathrm{MoO}_{3}$, and bathocuproine (BCP) were purchased from Luminescence Technology Corp. $\mathrm{CHNH}_{2} \mathrm{NH}_{2} \mathrm{I}$ (FAI) was purchased from Greatcell Solar. All materials were used as received.

\section{Device preparation}

ITO-coated glass substrates were subsequently cleaned with soap, water and isopropanol in an ultrasonic bath, followed by $20 \mathrm{~min}$ UV-ozone treatment. The substrates were transferred to a vacuum chamber integrated in a nitrogen-filled glovebox and evacuated to a pressure of $10^{-6} \mathrm{mbar}$ for the charge extraction layers' deposition. In general, the deposition rate for the TaTm and $\mathrm{C}_{60}$ was $0.5 \AA / \mathrm{s}$ while the thinner BCP layer was sublimed at $0.2 \AA / \mathrm{s} . \mathrm{MoO}_{3}$ and $\mathrm{Ag}$ were deposited in a second vacuum chamber using aluminum boats as sources, by applying currents ranging from 2.0 to $4.5 \mathrm{~A}$. The perovskite films were processed in a third, dedicated vacuum chamber. This is equipped with four evaporation sources (Creaphys) and with independent temperature controllers and shutters. All sources have an individual QCM sensor above an additional one is installed close to the substrates for the overall deposition rate measurement. All sources were individually calibrated for their respective materials and no cross-reading between different materials is ensured by the position of the sources, shutters, sensors. During the $\mathrm{FAMAPbI}_{3}$ perovskite deposition, the individual QCM deposition rates were: $1 \AA /$ s for FAI, $1 \AA / s$ for $\mathrm{PbI}_{2}$ and for MAI the rate was varied in the $0.7-1.9 \AA / \mathrm{s}$ range. During the perovskite deposition, the pressure of the chamber was below $8 \cdot 10^{-6}$ mbar and the substrates were kept at room temperature.

\section{Characterization}

Absorption spectra were collected using fiber optics based Avantes Avaspec2048 Spectrometer. The photoluminescence spectra were measured with an Avantes Avaspec2048 spectrometer and films were illuminated with a diode laser of integrated optics, emitting at $522 \mathrm{~nm}$. All the spectra were collected with an integration time of $1 \mathrm{~s}$. The crystalline structure of the powder and film samples was studied by X-ray diffraction (XRD). The patterns were collected in Bragg-Brentano geometry on an Empyrean PANalytical powder diffractometer with a copper anode operated at $45 \mathrm{kV}$ and $40 \mathrm{~mA}$. Further analysis including Le Bail fits were performed with Fullprof software. Scanning Electron Microscopy (SEM) images were performed on a Hitachi S-4800 microscope operating at an accelerating voltage of $2 \mathrm{kV}$ over platinum-metallized samples. 
The $J-V$ curves for the solar cells were recorded using a Keithley 2612A SourceMeter in a -0.2 and $1.2 \mathrm{~V}$ voltage range, with $0.01 \mathrm{~V}$ steps and integrating the signal for $20 \mathrm{~ms}$ after a $10 \mathrm{~ms}$ delay, corresponding to a scan speed of about $0.3 \mathrm{Vs}^{-1}$. The devices were illuminated under a Wavelabs Sinus 70 LED solar simulator. The light intensity was calibrated before every measurement using a calibrated Si reference diode. The external quantum efficiency (EQE) was estimated using the cell response at different wavelength (measured with a white light halogen lamp in combination with band-pass filters), where the solar spectrum mismatch was corrected with a calibrated Silicon reference cell (MiniSun simulator by ECN, from Netherlands).

For the sensitive EQE measurements, the cell was illuminated by a Quartz-Tungsten-Halogen lamp (Newport Apex 2-QTH) through a monochromator (Newport CS130-USB-3-MC), a chopper at $279 \mathrm{~Hz}$ and a focusing lens. The device current was measured as a function of energy from $2.1 \mathrm{eV}$ to $1.2 \mathrm{eV}$ in $0.02 \mathrm{eV}$ steps using a lock-in amplifier (Stanford Research Systems SR830). The system was calibrated and the solar spectrum mismatch was corrected using a calibrated Silicon reference cell.

Solar cell stability measurements were recorded using a maximum power point tracker (mppt) system, with a white LED light source under 1 sun equivalent, developed by Candlelight. During the mppt measurements, a flow of $\mathrm{N}_{2}$ gas was used and temperature was kept at $300 \mathrm{~K}$ using a water-circulating cooling system. 


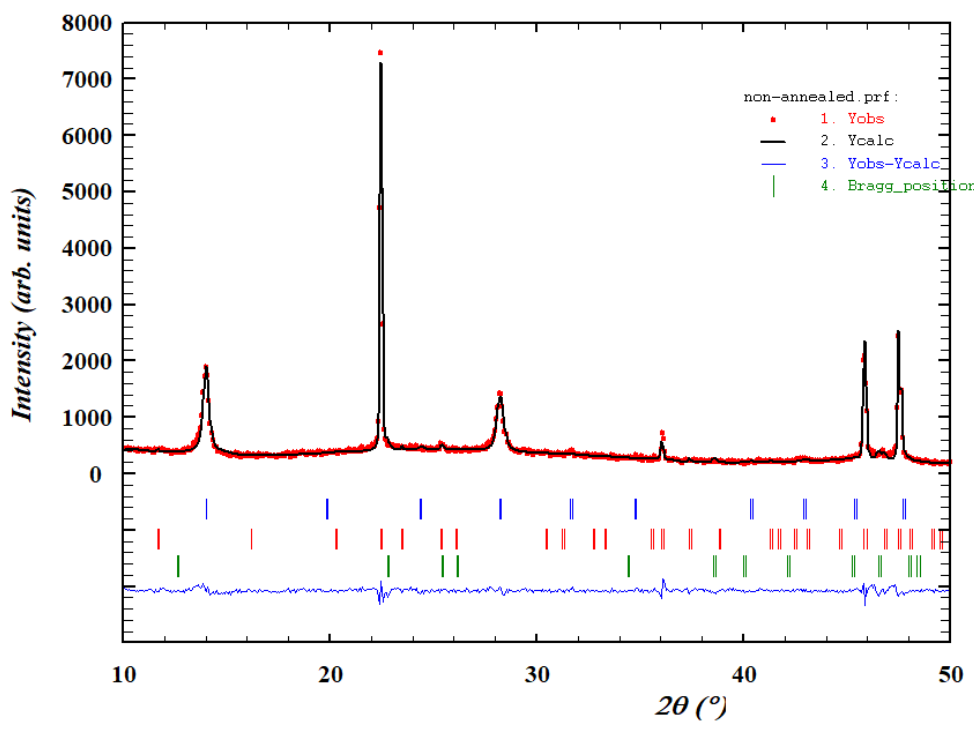

Figure S1. Whole-pattern Le Bail fit (black line) of XRD patterns (red dots) for as-deposited films from FAI:PbI ${ }_{2}$ co-deposition. Vertical markers correspond to calculated Bragg's reflections for $\alpha-\mathrm{FAPbI}_{3}$ perovskite (blue), yellow $\delta-\mathrm{FAPbI}_{3}$ phase (red), and $\mathrm{PbI}_{2}$ (green).

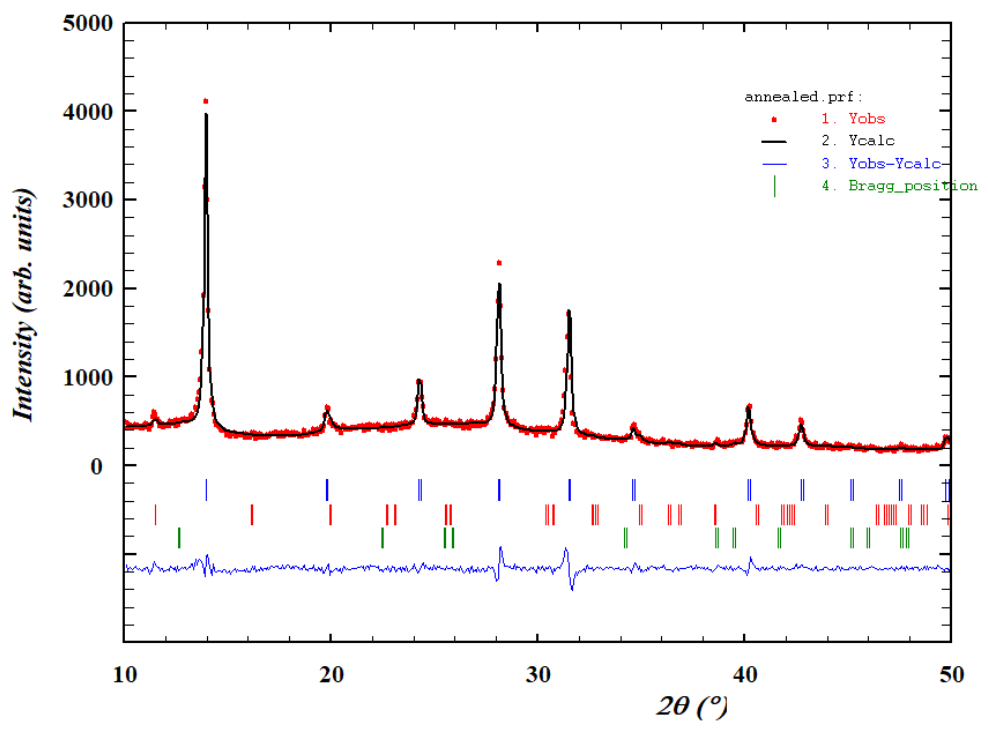

Figure S2. Whole-pattern Le Bail fit (black line) of XRD patterns (red dots) for $\mathrm{FAPbI}_{3}$ films annealed at $150{ }^{\circ} \mathrm{C}$ for 10 minutes. Vertical markers correspond to calculated Bragg's reflections for $\alpha-\mathrm{FAPbI}_{3}$ perovskite (blue), yellow $\delta-\mathrm{FAPbI}_{3}$ phase (red), and $\mathrm{PbI}_{2}$ (green). 

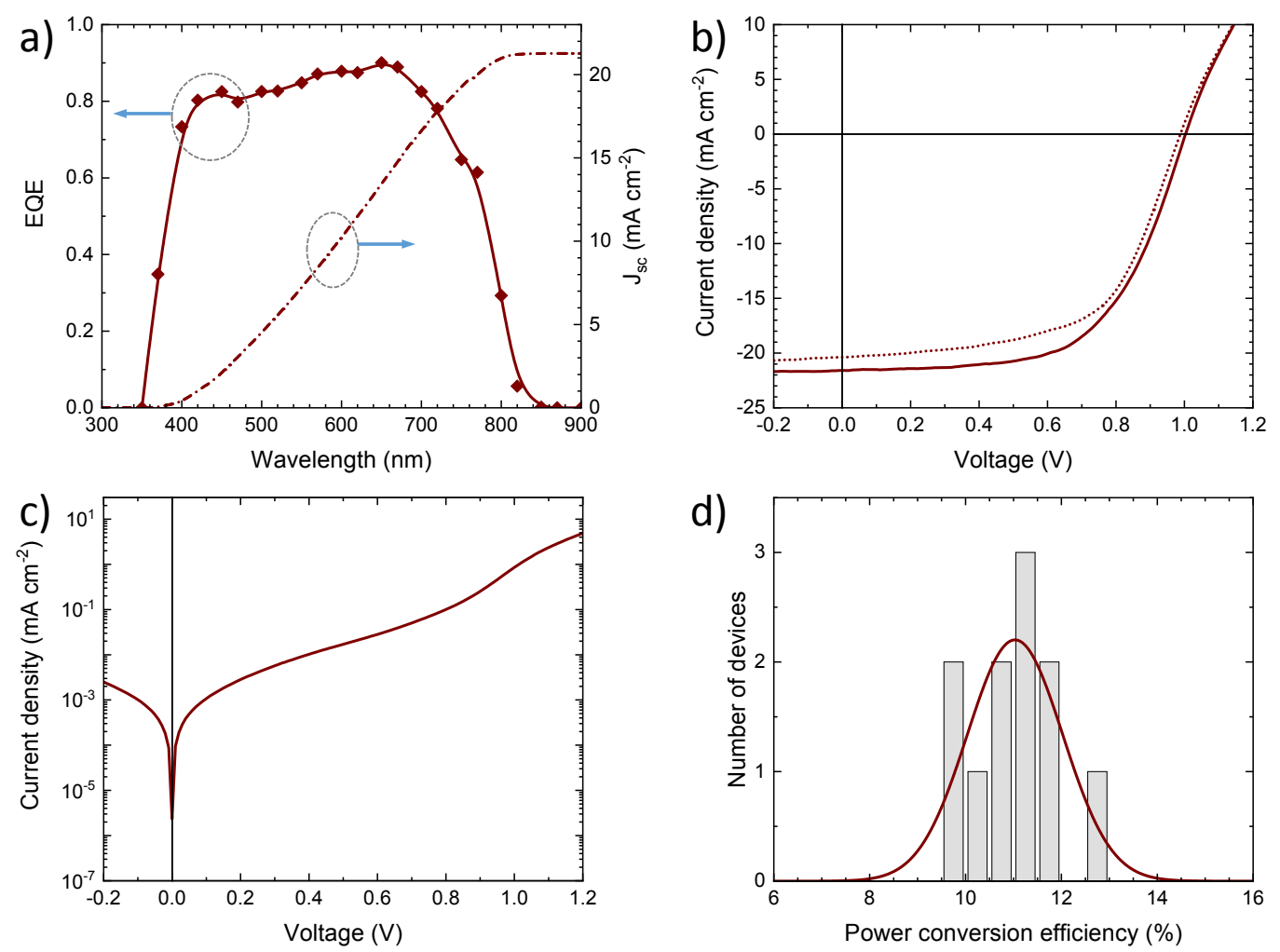

Figure S3. Characterization of solar cells using annealed $\mathrm{FAPbI}_{3}$ thin-film absorbers. (a) EQE spectrum (left axis) with integrated current over the solar AM1.5 spectrum (right axis), (b) J-V curves in forward (solid line) and reverse (dotted line) scans under illumination and (c) in the dark. (d) Statistics of the PCE for same devices.

Figure S4. In the following pages, the $\mathrm{XRD}$ characterization of $\mathrm{FAMAPbI}_{3}$ films deposited with increasing MAI deposition rates is reported as a function of the MAI deposition rate (indicated in each graph in $\AA / \mathrm{s}$ ). Whole-pattern Le Bail fit (red line) of XRD patterns (open black circles). Vertical markers correspond to calculated Bragg's reflections for cubic $\alpha$-phase perovskite (black), yellow $\delta$-phase (yellow), and $\mathrm{PbI}_{2}$ (green). 

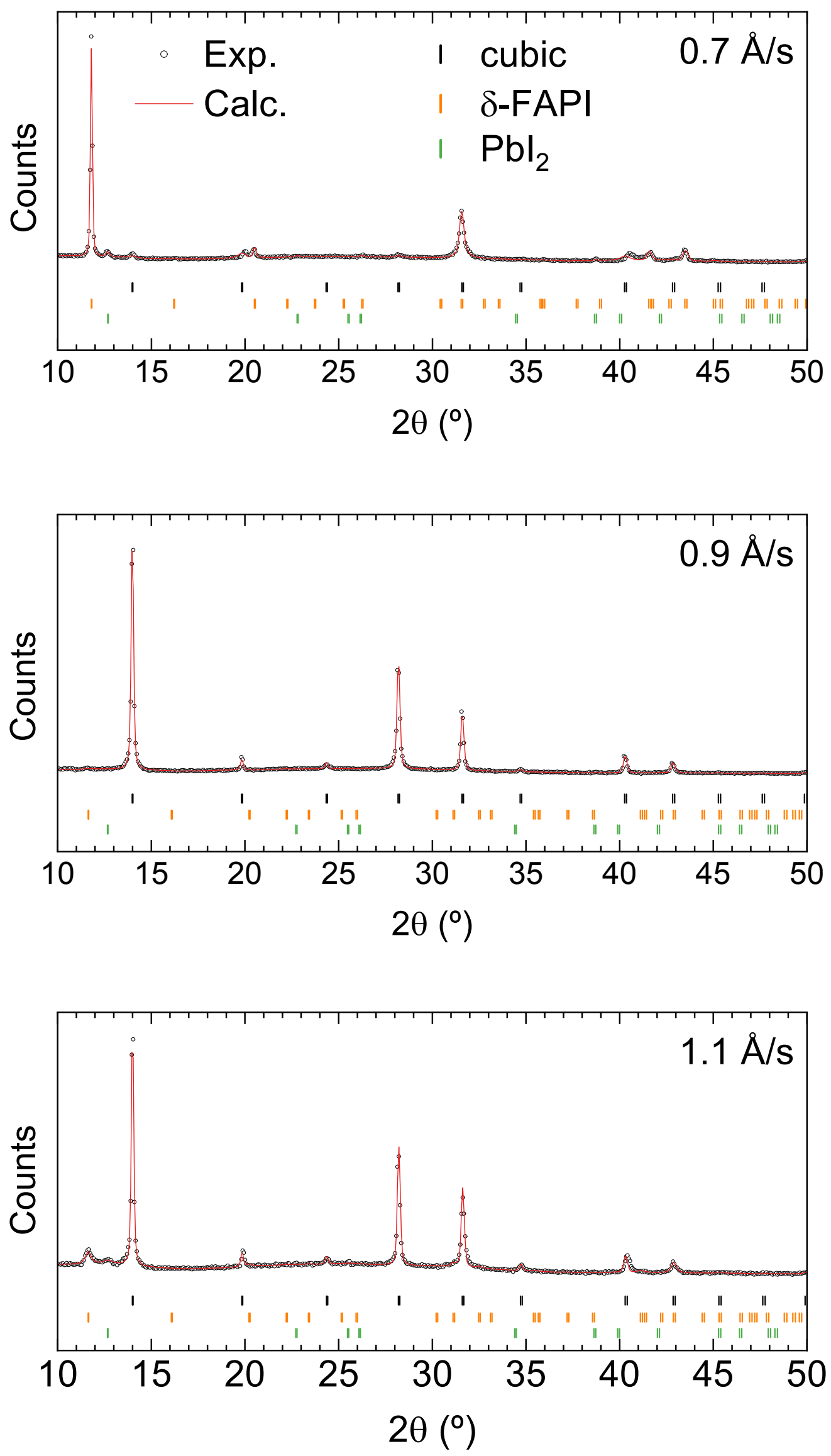

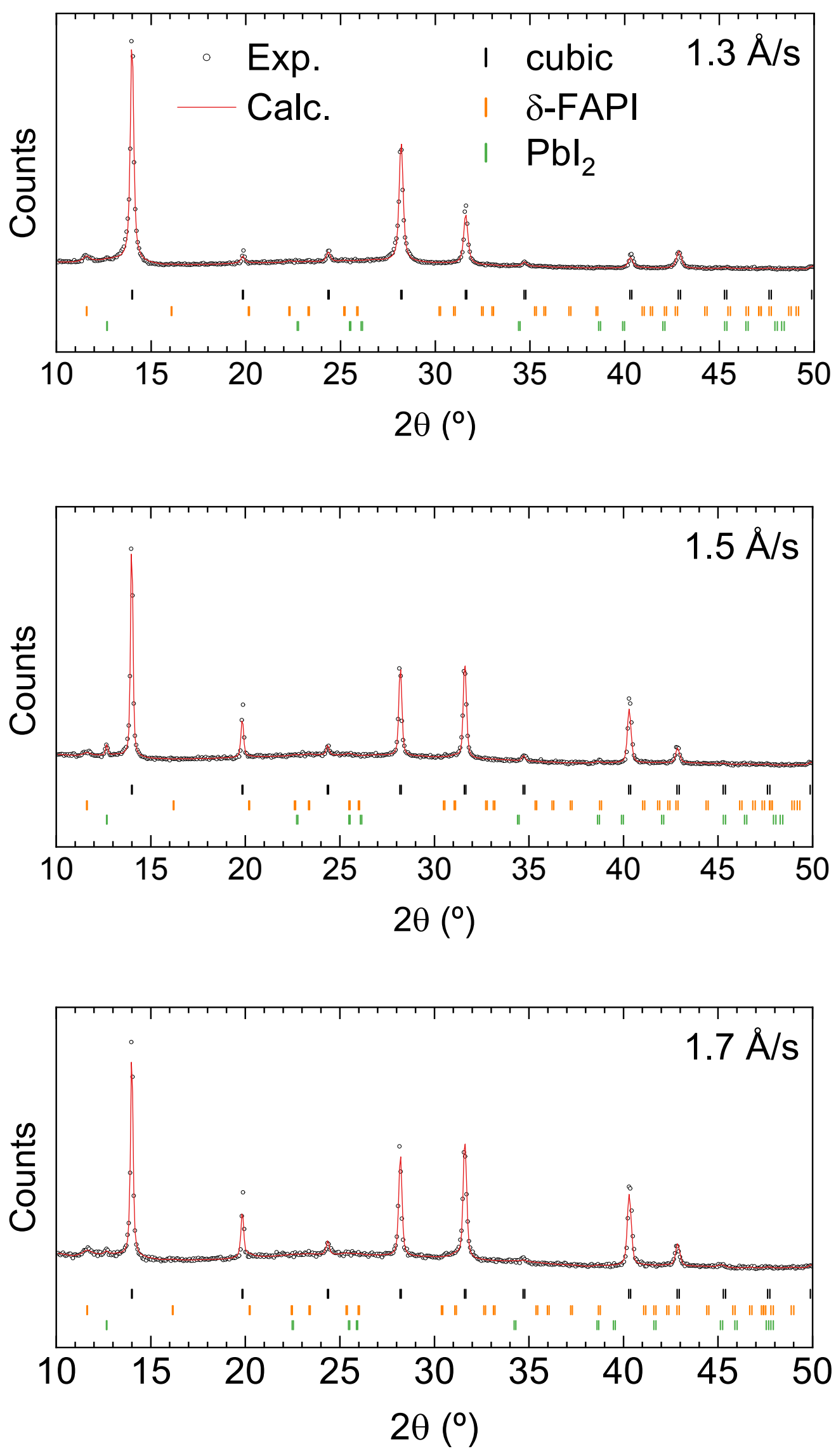

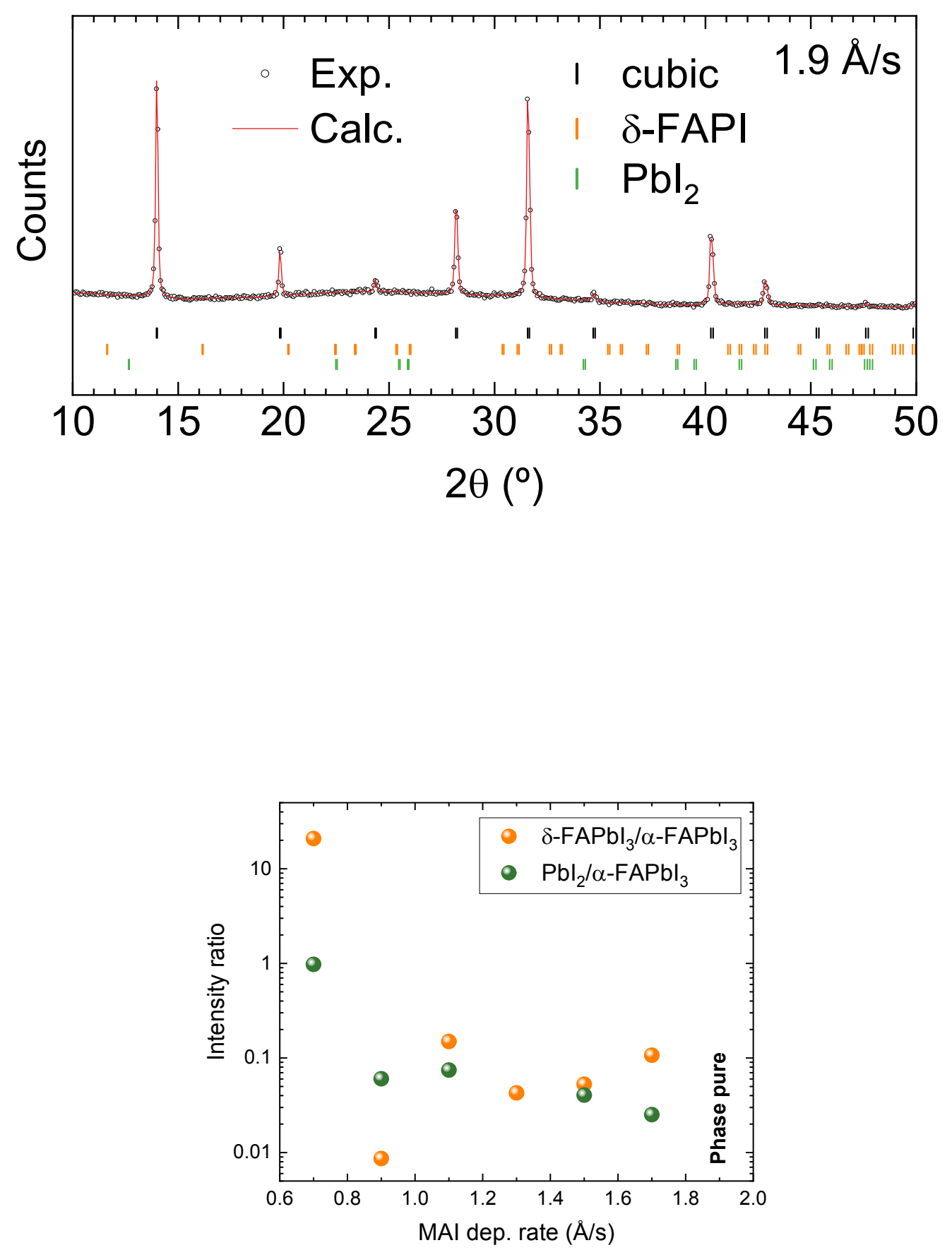

Figure S5. Semi-quantitative "yellow phase/black phase" and " $\mathrm{PbI}_{2} /$ black phase" ratios based on main diffraction peaks' calculated intensities for $\mathrm{FAMAPbI}_{3}$ films deposited at increasing MAI rate. 


\section{Determination of the film composition from quartz crystal microbalance (QCM) readings during perovskite deposition.}

The determination of the MAI deposition rate is not trivial as its adsorption is surface dependent. Hence, one can obtain the effective MAI deposition rate by analysis of the total deposition on the QCM close to the substrate (Figure S6a). However, this is only possible if the two other components are not surface dependent and can be monitored independently. For this reason, we employ 3 different sources and 1 substrate QCM (Figure S6a). In Figure S5b-c, the linear correlation between their respective source sensors and $\mathrm{S}-\mathrm{Sub}$, for $\mathrm{PbI}_{2}$ and FAI, respectively, is reported. This implies that we can monitor the sublimation rate of $\mathrm{FAI}$ and $\mathrm{PbI}_{2}$ independently from the relevant source QCM.

To establish if the FAI adsorption is not surface dependent we monitored the rate of FAI on $S$ $S u b$ with different $\mathrm{PbI}_{2}$ rates (ranging from 0 to $2 \AA / \mathrm{s}$ ). We can deduce the FAI rate on the $S$-Sub by subtracting the $\mathrm{PbI}_{2}$ rate from the total deposition rate at the $S$-Sub. The FAI rate on $S$-Sub was found to be constant regardless the rate of $\mathrm{PbI}_{2}$ (see the magenta line in Figure $\mathrm{S} 6 \mathrm{~d}$ ). Therefore, we can confirm that $\mathrm{FAI}$ and $\mathrm{PbI}_{2}$ possess surface independent adsorption characteristics.

Hence we can estimate the adsorbed thickness of MAI on the $S$-Sub by subtracting $\left(\mathrm{PbI}_{2}+\mathrm{FAI}\right)$ from the total $S-S u b$ thickness under the three source co-sublimation. In this way we take into account the surface dependent adsorption of MAI on the $S$-Sub during the 3 source co-deposition process.

One should be aware that $S-S u b$ is off-centered to prevent the shadows on the substrate by evaporated materials from the crucible sources. This results in different amount of molecules reaching the $S$-Sub and the substrate, depending on the location of the crucibles. To correct for this difference caused by the geometrical factor, we positioned two glass substrates: one is at the normal substrate holder and another is attached to the sensor head. Afterwards, the source materials are separately evaporated and the thickness of the evaporated films on the glass substrates is measured to calibrate the geometric differences. In our case, the ratio of the thickness on substrate/S-Sub were 1.3, 2.0, 2.2 for FAI, MAI and $\mathrm{PbI}_{2}$, respectively.

After considering all the above-mentioned precautions, we can deduce the composition from the QCM readings. The thickness on the QCM is directly proportional to the adsorbed mass, therefore, the molar ratio on the surface of $S$-Sub can be obtained by dividing by the respective molar mass of the source materials. Finally, the molar ratio for the fabricated films can be estimated by multiplying the geometric factors to the ratio obtained from the $S$-Sub. The results are summarized in Fig. S6e. 
(a)

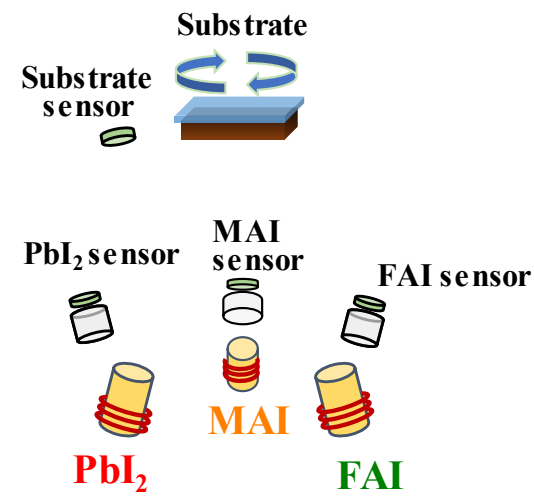

(c)

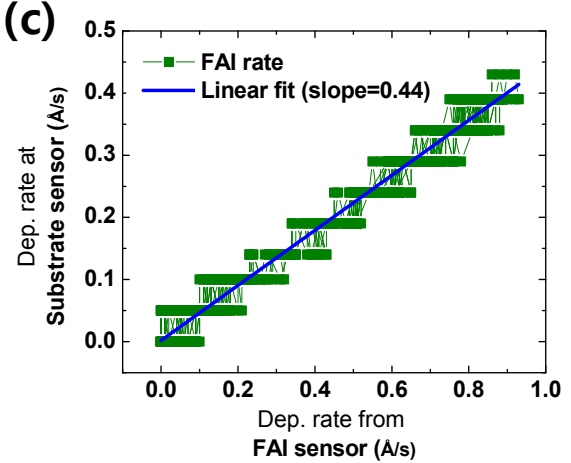

(e)

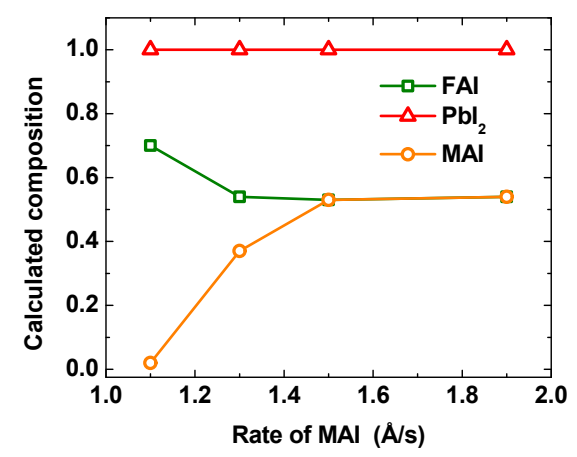

(b)

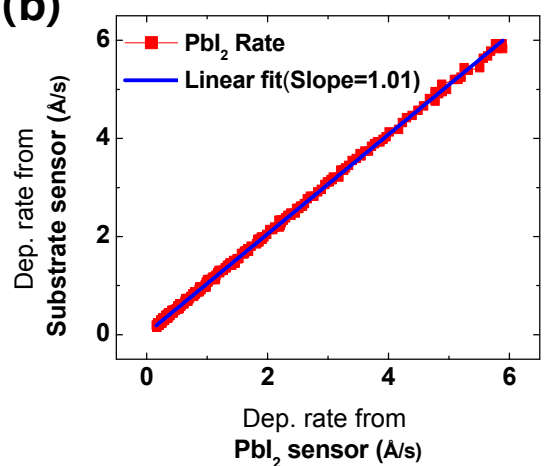

(d)

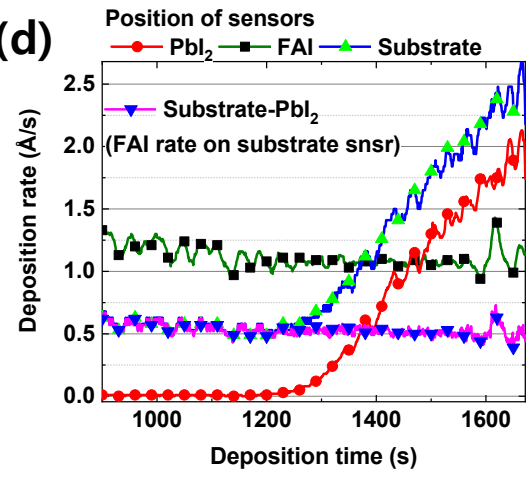

Figure S6 (a) Schematic illustration of the experimental set up used for the perovskite cosublimation. Deposition rates at source sensors and the substrate sensor for (b) $\mathrm{PbI}_{2}$ and (c) FAI. (d) Monitored deposition rates from the sensors with increased $\mathrm{PbI}_{2}$ rates under constant FAI rate (e) Calculated relative composition for the perovskite films based on the monitored thicknesses. 


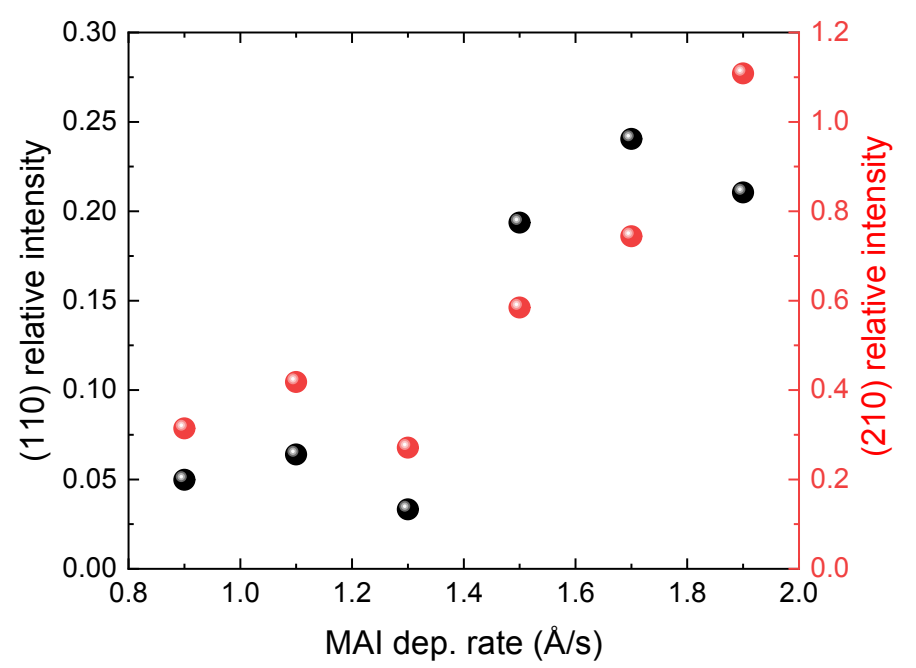

Figure S7. (110)/(100) and (210)/(100) calculated intensity ratios based on calculated diffraction pattern for $\mathrm{FAMAPbI}_{3}$ films deposited at increasing MAI rate. Note that calculated intensities from Le Bail fits take into account the multiplicity of the reflection. Hence, a value of 1 does not necessarily mean that the observed counts are equal for both peaks in the raw data.

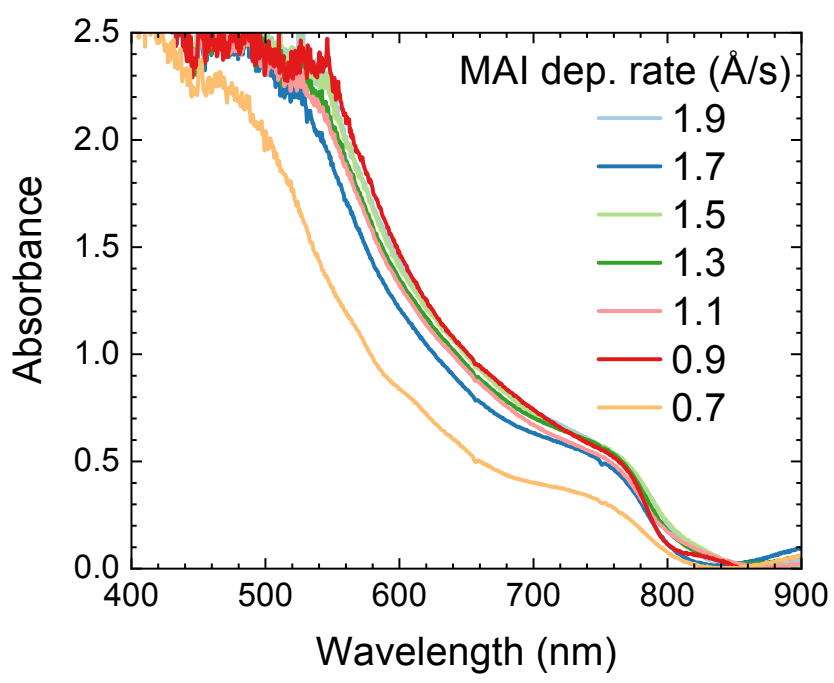

Figure S8. Optical absorption spectra for $\mathrm{FAMAPbI}_{3}$ perovskite films obtained with increasing MAI deposition rates. 

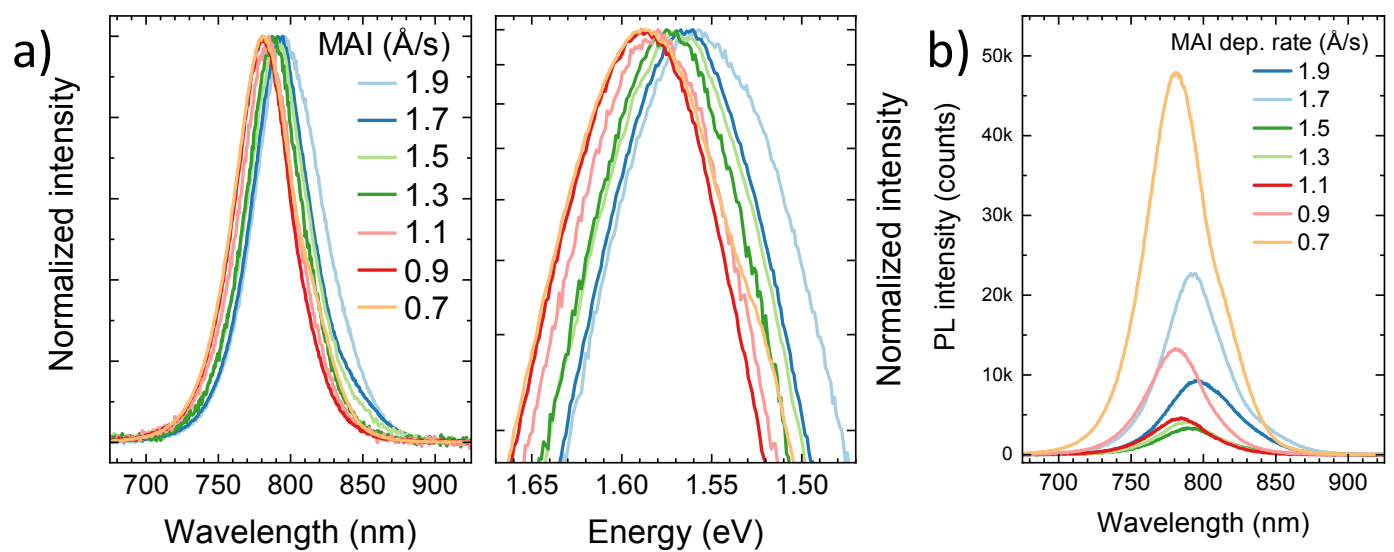

Figure S9. PL spectra for $\mathrm{FAMAPbI}_{3}$ perovskite films obtained with increasing MAI deposition rates. (a) Normalized spectra in linear (left) and log (right) scale, and (b) spectra without normalization. 
Table S1. PV performance parameters extracted from the J-V characteristics of the best $\mathrm{FAMAPbI}_{3}$ p-i-n solar cells with different MAI deposition rates.

\begin{tabular}{cccccc}
\hline $\begin{array}{c}\text { MAl rate } \\
(\AA / s)\end{array}$ & $\begin{array}{c}J_{s c} \\
\left(m A c^{-2}\right)\end{array}$ & $\begin{array}{c}J_{s c}(E Q E) \\
\left(m A c c^{-2}\right)\end{array}$ & $\begin{array}{c}F F \\
(\%)\end{array}$ & $\begin{array}{c}V_{o c} \\
(m V)\end{array}$ & $\begin{array}{c}P C E \\
(\%)\end{array}$ \\
\hline 0.7 & 18.0 & 17.2 & 71 & 1143 & 14.6 \\
0.9 & 22.0 & 21.5 & 69 & 1052 & 15.9 \\
1.1 & 22.3 & 21.9 & 74 & 1086 & 17.9 \\
1.3 & 22.1 & 22.2 & 76 & 1090 & 18.3 \\
1.5 & 22.6 & 22.5 & 76 & 1092 & 18.8 \\
1.7 & 22.2 & 21.8 & 75 & 1107 & 18.4 \\
1.9 & 21.5 & 22.3 & 64 & 1020 & 14.0 \\
\hline
\end{tabular}
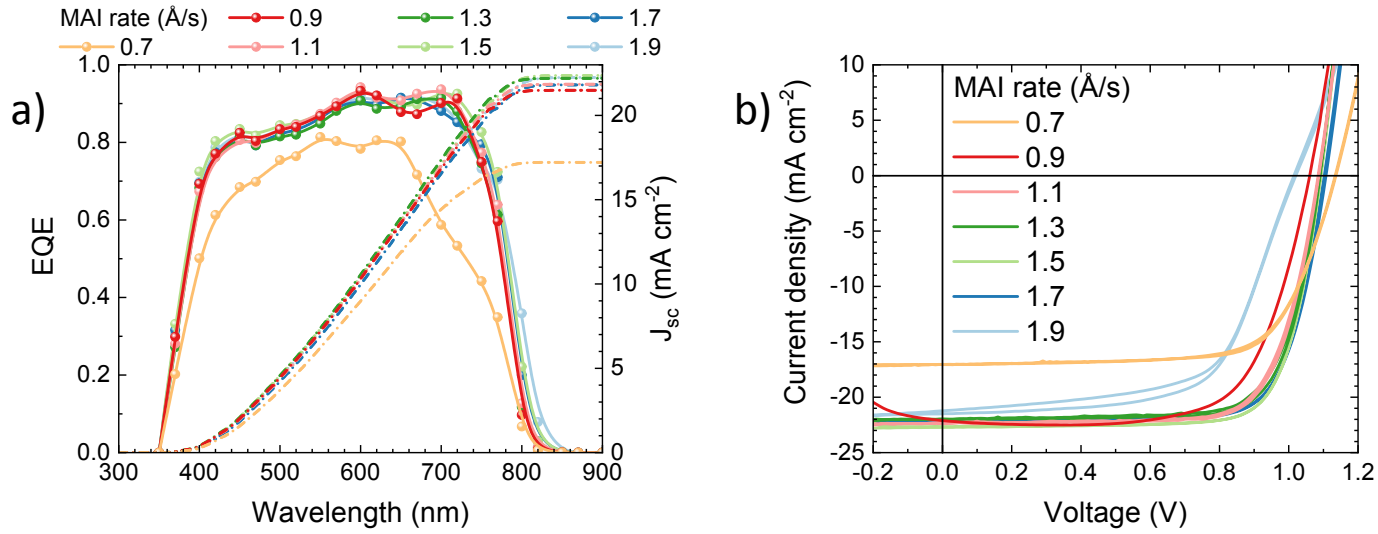

Figure S10. Characterization (a) External quantum efficiency (EQE) spectra. (b) J-V curves under illumination for perovskite solar cells with $\mathrm{FAMAPbI}_{3}$ absorbers obtained with varying MAI deposition rates during vacuum processing. 


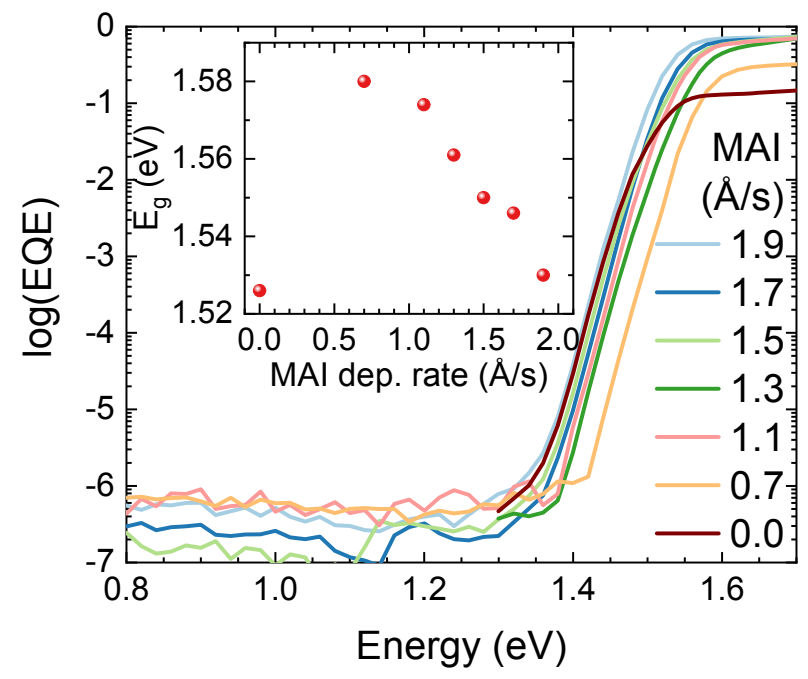

Figure S11. (a) Sensitive EQE spectra in the bandgap region (the inset show the calculated bandgap values for each MAI rate) of $\mathrm{FAMAPbI}_{3}$ solar cells prepared with increasing MAI deposition rates.

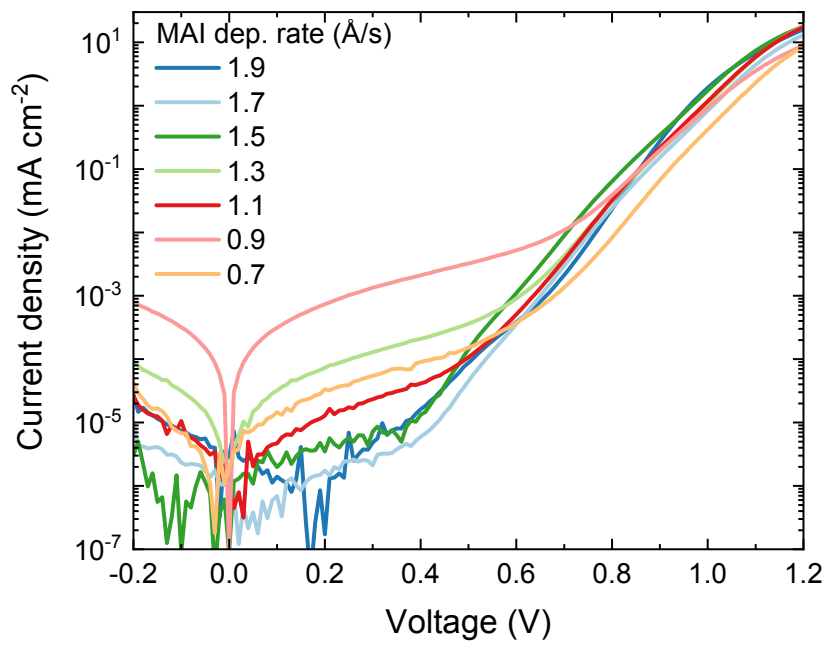

Figure S12. J-V curves collected in the dark for the series of $\mathrm{FAMAPbI}_{3}$ perovskite solar cells with varying MAI loading. 
Table S2. Bandgap and Urbach energies extracted form sensitive EQE analysis of the solar cells.

\begin{tabular}{ccc}
\hline $\begin{array}{c}\text { MAl rate } \\
(\AA / s)\end{array}$ & $\begin{array}{c}\text { Bandgap } \\
(\mathrm{meV})\end{array}$ & $\begin{array}{c}\text { Urbach Energy } \\
(\mathrm{meV})\end{array}$ \\
\hline 0.7 & 1.582 & 12.4 \\
1.1 & 1.574 & 12.7 \\
1.3 & 1.561 & 12.8 \\
1.5 & 1.554 & 12.9 \\
1.7 & 1.546 & 12.0 \\
1.9 & 1.533 & 12.8 \\
\hline
\end{tabular}

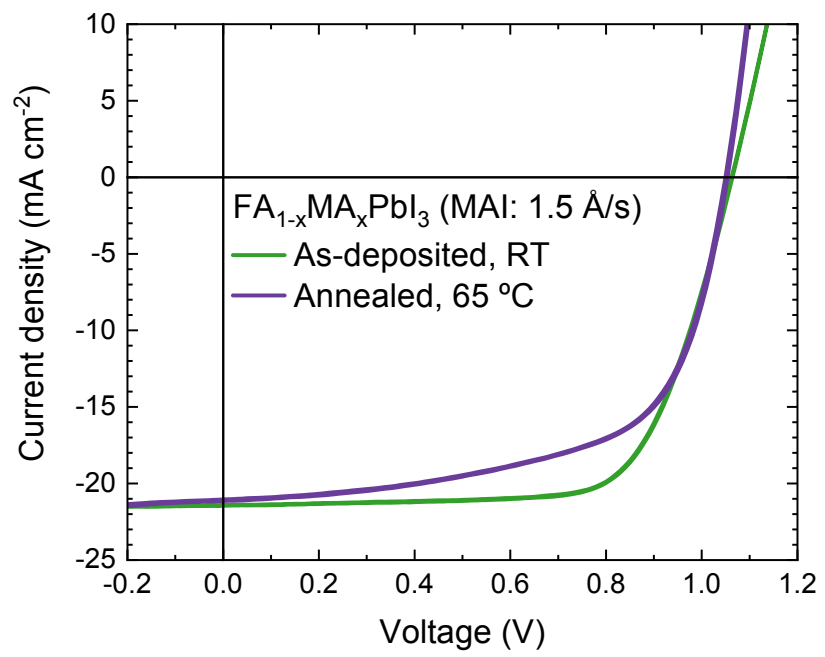

Figure S13. J-V curves under illumination for solar cells with as-prepared and annealed FAMAPbI $_{3}$ films obtained with MAI deposition rate of $1.5 \AA / \mathrm{s}$. 